\title{
MODELOS MATEMÁTICOS EM EPIDEMIOLOGIA E APLICAÇÃO NA EVOLUÇÃO DA COVID-19 NO BRASIL E NO ESTADO DA PARAÍBA
}

\author{
Célia Maria Rufino Franco ${ }^{1}$, Renato Ferreira Dutra ${ }^{2}$ \\ ${ }_{1}^{1}$ Professora da Unidade Acadêmica de Física e Matemática, Universidade Federal de Campina \\ Grande, Cuité, PB, Brasil. \\ ${ }^{2}$ Instituto de Física, Universidade Federal de Alagoas, Maceió-AL, Brasil. \\ E-mail para correspondência: celiarufino@ufcg.edu.br
}

\section{Resumo}

\begin{abstract}
Este trabalho tem como objetivo estudar alguns modelos matemáticos em Epidemiologia, sob o ponto de vista das aplicações, com particular referência a epidemia da Covid-19. São apresentados os modelos clássicos como SIS (Suscetível -Infectado - Suscetível), SIR (Suscetível - Infectado - Removido) e SIRS (Suscetível - Infectado- Removido - Suscetível), considerando a população total constante. Como aplicação, o modelo SIR foi utilizado para projetar a evolução epidêmica da Covid-19 no Brasil e no Estado da Paraíba. Para tanto, utilizou-se métodos numéricos (Euler ou Runge-Kutta) para resolver os sistemas de equações diferenciais e os parâmetros foram obtidos através de processo de otimização. De acordo com os resultados obtidos, o modelo descreve bem a população de infectados nos períodos analisados. Verificou-se mudança na taxa de reprodução $\left(R_{0}\right)$ da Covid-19 no Brasil, que passou de 3.7 (no início da epidemia) para 1.72 .
\end{abstract}

Palavras-chave: Modelagem Matemática, Covid-19, Brasil, Paraíba.

\begin{abstract}
This work aims to study some mathematical models in Epidemiology, from the point of view of applications, with particular reference to the Covid-19 epidemic. The classic models SIS (Susceptible - Infected - Susceptible), SIR (Susceptible Infected - Removed) and SIRS (Susceptible - Infected - Removed - Susceptible) are presented, considering the constant total population. As an application, the SIR model was used to project the epidemic evolution of Covid-19 in Brazil and in the State of Paraíba. For that, numerical methods were used (Euler or Runge-Kutta) to solve the systems of differential equations and the parameters were obtained through an optimization process. According to the results obtained, the model describes the infected population well in the analyzed periods. There was a change in the reproduction rate $\left(R_{0}\right)$ of Covid-19 in Brazil, which went from 3.7 (at the beginning of the epidemic) to 1.72 .
\end{abstract}

Keywords: Mathematical Modeling, Covid-19, Brazil, Paraíba. 


\section{Introdução}

De acordo com Rocha (2012), Epidemiologia é a ciência que estuda padrões da ocorrência de doenças em populações humanas e diferentes fatores que interferem na difusão e propagação de doenças e meios necessários à sua prevenção e erradicação. A prática clínica aborda a doença individualmente, enquanto a epidemiologia analisa a ocorrência de doenças em sociedade, ou seja, regiões, classes sociais, grupos específicos, entre outros.

Vários registros na história evidenciam impactos desastrosos de epidemias na humanidade. A peste negra foi uma das maiores pandemias já registradas, tendo início na China e se alastrando pela Europa durante o século XIV, matando cerca de um terço da população. Outras doenças também foram registradas tais como: cólera, tuberculose, varíola, gripe, sarampo e malária, que também causaram muitas mortes (LUIZ, 2012; ROCHA, 2012).

A Covid-19 é uma doença causada pelo vírus Sars-CoV-2, tendo sido registrado o primeiro caso oficial no dia 12 de dezembro de 2019 em Wuhan, China (GRUBER, 2020; WU, et al. 2020). Em dezembro de 2019, iniciou-se um surto contaminando cerca de 50 pessoas na cidade de Wuhan e espalhou-se pelo mundo, atingindo vários países. No Brasil, o primeiro caso reportado foi em 25 de fevereiro de 2020 de um morador da cidade de São Paulo que esteve na Itália (OLIVEIRA e ORTIZ, 2020).

Neste contexto, estudos começaram a ser realizados com o objetivo de compreender o desenvolvimento da doença em comunidades, regiões e países, e analisar o impacto de medidas de controle, como o desenvolvimento de vacina, ou outras medidas imprescindíveis para a sua contenção e erradicação (GONÇALVES, 2020; MORRISON et al., 2020; MANRIQUE-ABRIL et al., 2020).

Modelos matemáticos e simulações numéricas são ferramentas úteis para realizar projeções de como uma doença infecciosa se propaga e testar teorias e conjecturas de avaliação quantitativa. Com os modelos matemáticos é possível determinar parâmetros e projetar a evolução de epidemias, como por exemplo da Covid-19. Inicialmente os parâmetros são estimados com base no conhecimento acumulado em epidemias anteriores ou da própria epidemia e do seu desenvolvimento em outros locais. Os modelos compartimentais do tipo 
SIR (suscetível-infectado-removido) e do tipo SIRS (suscetível-infectadoremovido-suscetível) foram desenvolvidos por Kermack e McKendrick, em 1927 e 1933, respectivamente, para descrever infecções endêmicas (ROCHA, 2012).

Luiz (2012) realizou um estudo dos modelos SIS, SIR e SIRS através da análise de estabilidade de pontos de equilíbrio dos sistemas de equações diferenciais envolvidos e apresenta uma variação do modelo SIR para a gripe Influenza A H1N1.

Tavares (2017) mostra uma aplicação do modelo SIR para descrever um surto de gripe em uma escola. Foi possível verificar como a matemática pode ser útil na previsão de uma epidemia e na tomada de decisão sobre estratégias de combate à sua propagação (vacinação, quarentena, etc.).

Em MANRIQUE-ABRIL et al. (2020) é apresentado um estudo da pandemia da Covid-19 na Colômbia baseada no modelo SIR. Os resultados mostram a taxa de reprodução da doença que foi igual a 2 nos primeiros 15 dias e o pior cenário aconteceu na primeira semana de abril de 2020, com taxa de reprodução igual a 3 .

LYRA et al. (2020) avaliaram a viabilidade do isolamento vertical como estratégia para o Brasil. Foi desenvolvido um modelo SEIR modificado, incluindo confinamento, transmissão assintomática, quarentena e hospitalização. A população foi subdividida em 9 faixas etárias, resultando em um sistema de 72 equações diferenciais não lineares acopladas. A estratégia testada é do confinamento de indivíduos com mais de 60 anos e a liberação total da população mais jovem a partir de $1^{\circ}$ de maio de 2020. O estudo mostra que esse tipo de estratégia resultaria em 400.000 internações, 50.000 casos em UTI e 120.000 mortes na faixa etária de 50 a 60 anos. O sistema de saúde evita o colapso se a população entre 50 e 60 anos de idade também permacesse em isolamento. Os resultados desencorajam o confinamento por idade como estratégia de saída.

Gonçalves et al. (2020) apresentam modelagem matemática do Covid-19 no Brasil, para o Estado do Rio Grande do Sul e para a Cidade de Porto Alegre. O estudo mostra a aplicação do modelo SIR na evolução do Covid-19. Para o Brasil considerou-se a taxa de reprodução da doença como sendo 3.2 
até 8 dias depois do caso 50, 2.08 de 9 a 18 dias, 1.72 de 18 a 30 dias e 1.4 para o restante dos dias.

Neste artigo, apresentar-se-á três modelos matemáticos clássicos em Epidemiologia: SIS, SIR e SIRS com o objetivo de mostrar a grande aplicabilidade da modelagem matemática em problemas reais. Procura-se responder os seguintes questionamentos: Em que condições tem-se uma epidemia? Por que uma epidemia aparentemente desaparece depois de um certo número de pessoas serem contaminadas? Quando o número de infectados será decrescente? Qual a taxa de infecção da doença?

Como aplicação do modelo SIR, resultados da simulação da expansão da Covid-19 no Brasil e no Estado da Paraíba são apresentados e analisados.

\section{Modelos Compartimentais: Modelos SIS, SIR e SIRS}

Nos modelos compartimentais, a população é dividida em compartimentos (ou classes) que refletem o estado em que os indivíduos se encontram no desenvolvimento da doença, como por exemplo:

- Suscetíveis (S): indivíduos que estão suscetíveis a contrair a doença;

- Infectados (I): indivíduos que contraíram a doença e podem transmiti-la aos indivíduos suscetíveis por transmissão direta;

- Removidos (R): indivíduos que foram infectados, mas não são mais portadores da doença, por motivo de isolamento, cura (adquirindo ou não imunidade), ou morte.

Assim, pode-se escrever a população total $\mathrm{N}$ como a soma dos indivíduos das classes acima citadas, ou seja, $\mathrm{N}=\mathrm{S}+\mathrm{I}+\mathrm{R}$. Neste caso, $\mathrm{N}$ é constante, isto é, não é considerado natalidade e mortalidade devido a outros fatores.

\subsection{Modelo SIS}

No modelo SIS, os indivíduos suscetíveis adquirem a doença, tornam-se infectados e, após a recuperação, não adquirem imunidade, tornando-se suscetíveis novamente. O período de incubação é relativamente pequeno e as pessoas doentes não são isoladas. É chamado modelo SIS uma vez que o 
caminho típico da transmissão da doença é de $S$, passando por I, até $S$ novamente. Considere o caso em que a população total N é constante e não há dinâmica vital, isto é, na população estudada não são considerados nascimentos nem mortes.

O modelo envolve dois parâmetros importantes: $\alpha$ e $\beta$, onde $\alpha$ é a taxa de transmissão e $\beta$ a taxa de recuperação da doença. Como essa transmissão se dá com o contato entre suscetíveis e infectados, então a variação de indivíduos suscetíveis em relação ao tempo pode ser modelada por $\alpha$ SI. Considerando que a variação dos indivíduos infectados com relação ao tempo é proporcional ao próprio número de indivíduos infectados, então o retorno à classe de suscetíveis será modelado por $\beta I$ (LUIZ, 2012).

Observe que uma fração dos indivíduos suscetíveis, por meio do contato com os indivíduos infectados, adquirem a doença e passam para a classe de infectados. Do mesmo modo, os indivíduos infectados, ao se recuperarem, não adquirem imunidade e retornam à classe de suscetíveis, como mostra a Figura 1.

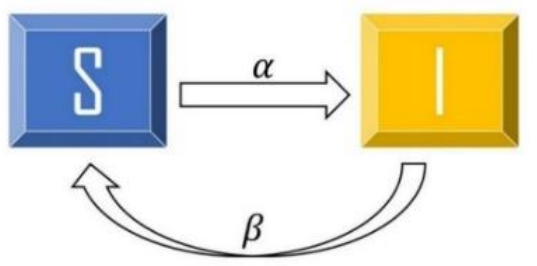

Figura 1: Representação do modelo SIS

Fonte: Autores

A dinâmica de uma doença com essas características pode ser descrita pelo sistema de equações diferenciais (BASSANEZI et al., 1988; LUIZ, 2012; TAVARES, 2017):

$$
\left\{\begin{array}{l}
\frac{d S}{d t}=-\alpha S I+\beta I \\
\frac{d I}{d t}=\alpha S I-\beta I
\end{array}\right.
$$

onde $\alpha, \beta>0$ e $\mathrm{N}=\mathrm{S}(\mathrm{t})+\mathrm{I}(\mathrm{t})$ com condições iniciais $\mathrm{I}(0)=\mathrm{I}_{0}$ e $\mathrm{S}(0)=\mathrm{S}_{0}=$ 
$\mathrm{N}-\mathrm{I}_{0}$.

Seja $R_{0}=\alpha S / \beta$ a taxa de reprodução básica que significa o número médio de infecções causadas por um indivíduo doente, onde $1 / \beta$ é o tempo médio no qual um indivíduo permanece infectado e $\alpha \mathrm{S}$ é a taxa de propagação da doença provocada pela introdução de um indivíduo infectado numa população de suscetíveis.

Observe que:

- Se $\mathrm{R}_{0}>1$ e $\mathrm{I} \neq 0$, então $\mathrm{dI} / \mathrm{dt}>0$ e $\mathrm{dS} / \mathrm{dt}<0$. O que significa que a epidemia alastra-se pela população.

- Se $\mathrm{R}_{0}<1$ e $\mathrm{I} \neq 0$, então $\mathrm{dI} / \mathrm{dt}<0$ e $\mathrm{dS} / \mathrm{dt}>0$. O que significa que $\mathrm{O}$ contágio diminui.

- Se $\mathrm{I}=0$, então $\mathrm{N}=\mathrm{S}$ e todas as pessoas são saudáveis, ou seja, não existe infecção.

Neste caso, os pontos de equilíbrio do sistema são $(\mathrm{S}, \mathrm{I})=(\beta / \alpha, \mathrm{N}-\beta / \alpha)$ e $(S, I)=(N, 0)$. Note que $-d S / d t=d I / d t=\alpha I(S-\beta / \alpha)$, de onde obtém-se as seguintes conclusões:

- Se I $=0$ ou $S=\beta / \alpha$, então $\mathrm{dS} / \mathrm{dt}=0$ e $\mathrm{dI} / \mathrm{dt}=0 \mathrm{e}$, portando, não há epidemia.

- Se $S=\beta / \alpha$, então $I=N-\beta / \alpha$ e, neste caso, $-d S / d t=d I / d t=0$.

- Se $I \neq 0$ e $S>\beta / \alpha$ ou $S<\beta / \alpha$, então o ponto $(S, I) \rightarrow(\beta / \alpha, N-\beta / \alpha)$ sobre a reta $\mathrm{S}=\mathrm{N}-\mathrm{I}$. Neste caso, o ponto $(\beta / \alpha, \mathrm{N}-\beta / \alpha)$ é assintoticamente estável, o que significa que a epidemia estabiliza-se nesse ponto.

O ponto de equilíbrio $(\mathbf{S}, \mathbf{I})=(\mathbf{N}, \mathbf{0})$ é instável, pois as soluções não constantes se afastam desse ponto. Se $\mathbf{I} \neq \mathbf{0}$, por menor que seja, a doença se propaga.

A maior velocidade da epidemia é obtida quando $\frac{\mathrm{d}}{\mathrm{dt}}$ atinge seu ponto máximo, isto é, quando $\frac{\mathrm{d}^{2} \mathrm{I}}{\mathrm{dt}^{2}}=0$. Assim, a curva de infectados tem um ponto de inflexão em

$$
\mathrm{I}^{*}=\frac{1}{2}\left(\mathrm{~N}-\frac{\beta}{\alpha}\right)
$$


ou seja, no ponto médio entre os dois pontos de equilíbrio ( $\mathrm{I}=0$ e $\mathrm{I}=\mathrm{N}-\beta / \alpha$ ). A partir do ponto de inflexão a curva muda de concavidade e a epidemia tende a estabilizar-se.

\subsection{Modelo SIR}

A suposição básica deste tipo de modelo é que um indivíduo pode passar sucessivamente por estágios de suscetibilidade (S), infecção (I) e removido (R) porque recuperou e adquiriu imunidade permanente, isto é, durante toda a vida; ou acabou morrendo. Considerando o período de incubação relativamente pequeno e a população constante (contando também com os mortos devido a infecção), tem-se: $\mathrm{N}=\mathrm{S}(\mathrm{t})+\mathrm{I}(\mathrm{t})+\mathrm{R}(\mathrm{t})$. A figura 2 mostra a representação do modelo SIR.

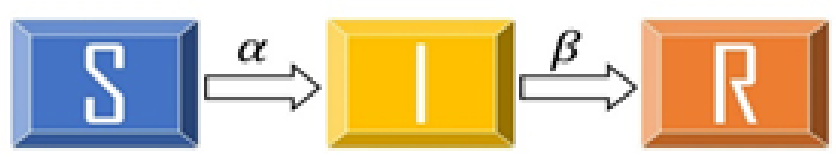

Figura 2: Representação do modelo SIR

Fonte: Autores

Levando-se em conta que a variação da população recuperada é proporcional à população infectada, o sistema de equações diferenciais que descreve a dinâmica desta epidemia é dado por (BASSANEZI et al., 1988; LUIZ, 2012):

$$
\left\{\begin{array}{l}
\frac{\mathrm{dS}}{\mathrm{dt}}=-\alpha \mathrm{IS} \\
\frac{\mathrm{dI}}{\mathrm{dt}}=\alpha \mathrm{IS}-\beta \mathrm{I} \\
\frac{\mathrm{dR}}{\mathrm{dt}}=\beta \mathrm{I}
\end{array}\right.
$$

onde $\alpha>0$ é o coeficiente de transmissão que determina a taxa a que novas infecções surgem como consequência do contato entre indivíduos suscetíveis e infectados, e $\beta>0$ denota a taxa de recuperação. Com as condições iniciais $\mathrm{R}(0)=\mathrm{R}_{0}=0, \mathrm{I}(0)=\mathrm{I}_{0}$ e $\mathrm{S}(0)=\mathrm{S}_{0}=\mathrm{N}-\mathrm{I}_{0}$. 
Uma epidemia cresce se o número de indivíduos infectados aumenta. Isto é, $\mathrm{dI} / \mathrm{dt}>0$ e I $\neq 0$. Assim,

$$
\frac{\mathrm{dI}}{\mathrm{dt}}>0 \Leftrightarrow \alpha \mathrm{SI}-\beta \mathrm{I}>0 \Leftrightarrow \frac{\alpha \mathrm{S}}{\beta}>1
$$

e

$$
\frac{\mathrm{dI}}{\mathrm{dt}}<0 \Leftrightarrow \alpha \mathrm{SI}-\beta \mathrm{I}<0 \Leftrightarrow \frac{\alpha \mathrm{S}}{\beta}<1
$$

Seja $\mathrm{R}_{0}=\alpha \mathrm{S} / \beta$ a taxa de reprodução que significa o número médio de infecções causadas pela inserção de um indivíduo doente sobre uma população sem imunidade à doença e na ausência de qualquer controle, onde $\frac{1}{\beta}$ é o tempo médio no qual um indivíduo permanece infectado e $\alpha \mathrm{S}$ é a taxa de propagação da doença provocada pela introdução de um indivíduo infectado numa população de suscetíveis. Logo, se $\mathrm{R}_{0}>1$ a epidemia permanecerá na população. Se $\mathrm{R}_{0}<1$ a doença desaparece.

Quanto maior for a taxa de remoção (recuperação) relativamente à taxa de infecção, mais rapidamente a epidemia cessará. Conhecendo $R_{0}$ e $\beta$, podese determinar a taxa de infecção $\alpha$.

Das duas primeiras equações do sistema (3), tem-se que I $=0$ é ponto de equilíbrio. Como $\frac{\mathrm{d} S}{\mathrm{dt}}<0$, então $S$ é sempre decrescente. Quando $t$ cresce, o ponto $(S, I)$ move-se ao longo da trajetória no plano de fase SI, com S sempre decrescendo, I cresce se $S_{0}>\frac{\beta}{\alpha}$ e decresce para zero se $S_{0}<\frac{\beta}{\alpha}$.

O sistema (3) é um sistema não-linear. Mas, uma vez que as duas primeiras equações só dependem de S e I, é possível reduzi-las a uma única equação:

$$
\frac{\mathrm{dI}}{\mathrm{dS}}=\frac{\alpha \mathrm{SI}-\beta \mathrm{I}}{-\alpha \mathrm{SI}}=\frac{\beta}{\alpha \mathrm{S}}-1
$$

A Equação (6) pode ser resolvida por separação de variáveis, obtendo-se 


$$
\mathrm{I}=-\mathrm{S}+\frac{\beta}{\alpha} \ln \left(\frac{\mathrm{S}}{\mathrm{S}_{0}}\right)+\mathrm{K}
$$

Usando as condições iniciais, determina-se o valor de $K=N-\frac{\beta}{\alpha} \ln \left(S_{0}\right)$. Assim,

$$
\mathrm{I}=\mathrm{N}-\mathrm{S}+\frac{\beta}{\alpha} \ln \left(\frac{\mathrm{S}}{\mathrm{S}_{0}}\right)
$$

Da Equação (8), tem-se que $\mathrm{I} \rightarrow-\infty$ quando $\mathrm{S} \rightarrow 0$, e como $\mathrm{I}_{0}>0$, existe pelo menos um valor de $S$ para o qual $\mathrm{I}=0$. Em outras palavras, uma epidemia acaba antes de todos os indivíduos suscetíveis se infectarem.

\subsection{Modelo SIRS}

Neste modelo, há indivíduos recuperados que perdem a imunidade, após certo período de tempo, voltando a ser suscetíveis. Consideraremos a população total $\mathrm{N}$ constante e que não há dinâmica vital. Seja $\delta$ a taxa de perda de imunidade. Então, como a perda de imunidade é proporcional ao número de indivíduos removidos, a modelagem é dada por $\delta \mathrm{R}$. $\mathrm{O}$ fluxo de transmissão é da classe $\mathrm{S}$ à classe $\mathrm{I}$, depois para a classe $R$ e, por fim, devido à não imunidade do individuo, de volta à classe $S$, como mostra a Figura 3.

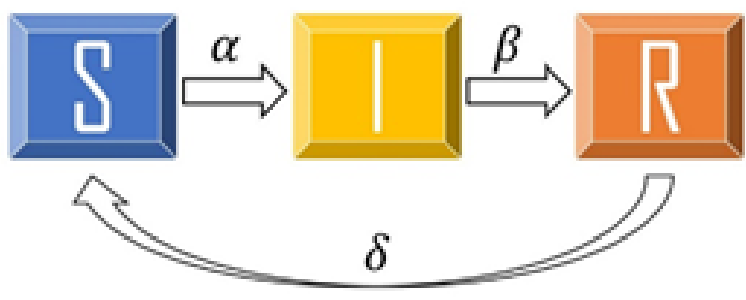

Figura 3: Representação do modelo SIR

Fonte: Autores

Assim, o sistema de equações diferenciais que representa essa dinâmica é da forma (BASSANEZI et al., 1988; ROCHA, 2012): 


$$
\left\{\begin{array}{l}
\frac{d S}{d t}=-\alpha S I+\delta R \\
\frac{d I}{d t}=\alpha S I-\beta I \\
\frac{d R}{d t}=\beta I-\delta R
\end{array}\right.
$$

onde, $\alpha, \beta, \delta>0, \alpha I$ é a taxa de infecção, $\beta$ denota a proporção de pessoas que deixaram a classe dos infectados para a classe de recuperados/removidos. $\mathrm{O}$ número total da população é dado por $\mathrm{N}=\mathrm{S}+\mathrm{I}+\mathrm{R}$, que é um valor constante. Com as condições iniciais $\mathrm{R}(0)=\mathrm{R}_{0}=0, \mathrm{I}(0)=\mathrm{I}_{0}$ e $\mathrm{S}(0)=\mathrm{S}_{0}=$ $\mathrm{N}-\mathrm{I}_{0}$.

\section{$3 \quad$ Resultados e discussões}

Projeções da COVID-19 no Brasil e no Estado da Paraíba vem sendo apresentada baseada no modelo SIR. Mesmo com as medidas de isolamento social adotadas, que reduz a exposição independente de terem sintomas, e a obrigatoriedade da quarentena para indivíduos infectados que evita a transmissão, tem-se que essas medidas não são observadas imediatamente no número de casos, pois existe uma defasagem entre o momento da infecção e o aparecimento de sintomas (período de incubação). O tempo de incubação da COVID-19 é de 5 a 14 dias (tendo referências que citam 5 a 18 e 5 a 21 dias), como é reportado pelo grupo de pesquisadores do Laboratório do Grupo de Dispersão de Poluentes \& Engenharia Nuclear (GDISPEN), disponível em Gonçalves et al. (2020).

Neste trabalho, considerou-se um período (tempo médio) de 10 dias que um indivíduo infectado se mantém em circulação (taxa de recuperação $\beta=\frac{1}{10}$ ). Reescrevendo o sistema (3) na forma

$$
\left\{\begin{array}{l}
\frac{\mathrm{d} S}{\mathrm{dt}}=-\alpha \mathrm{S} \frac{\mathrm{I}}{\mathrm{N}} \\
\frac{\mathrm{dI}}{\mathrm{dt}}=\alpha \mathrm{S} \frac{\mathrm{I}}{\mathrm{N}}-\beta \mathrm{I} \\
\frac{\mathrm{dR}}{\mathrm{dt}}=\beta \mathrm{I}
\end{array}\right.
$$


a taxa de transmissão da doença $\alpha$ foi obtida, utilizando-se um código computacional, através de processo de otimização até que os resultados simulados para o número de infectados possam ser considerados suficientemente próximos aos dados fornecidos pelo Ministério da Saúde (MS). A correlação do modelo com os dados de infectados fornecidos pelo MS será tanto mais forte quanto mais próximo o coeficiente de determinação $R_{d}^{2}$ estiver de 1.

Foi possível obter a taxa de reprodução básica $\mathrm{R}_{0}=\frac{\alpha}{\beta}$, que mede a velocidade com que a epidemia se propaga. Medidas como distanciamento social e quarentena tem o efeito de diminuir a taxa de infecção $\alpha$, e consequentemente $\circ \mathrm{R}_{0}$.

A Figura 4 mostra a comparação entre os dados simulados e os dados oficiais fornecidos pelo MS (Ministério da Saúde) para os infectados no Brasil no período de 25/02/2020 a 23/03/2020 (BRASIL, 2020). Os dados dos casos confirmados foram extraídos da página: https://covid.saude.gov.br/ (acesso em 03/05/2020). A Tabela 1 mostra o parâmetro de entrada e o parâmetro ajustado. Neste caso, o coeficiente de determinação obtido foi $R_{d}^{2}=$ 0.996648215 .

Tabela 1: Parâmetros usados no modelo SIR para Brasil

\begin{tabular}{|c|c|c|c|c|}
\hline \multicolumn{2}{|c|}{ Período População (N) } & $\begin{array}{c}\mathrm{I}_{0}\left(\mathrm{n}^{\mathrm{o}} \text { de }\right. \\
\text { infectados } \\
\text { inicial }\end{array}$ & $\beta\left[\right.$ Dias $\left.^{-1}\right]$ & $\begin{array}{c}\alpha\left[\mathrm{Dias}^{-1}\right] \\
\text { (Parâmetro } \\
\text { ajustado) }\end{array}$ \\
\hline $\begin{array}{c}25 / 02 / 2020 \mathrm{a} \\
23 / 03 / 2020\end{array}$ & 210.000 .000 & 1 & 0.1 & 0.370057653 \\
\hline
\end{tabular}

Fonte: Autores

Neste período analisado, a taxa de reprodução da doença é $\mathrm{R}_{0}=$ 3.700576528, que significa o número médio de pessoas que são infectadas por um único indivíduo. $\mathrm{O}$ valor de $\mathrm{R}_{0}$ é compatível com o obtido em GONÇALVES, et al. (2020). 


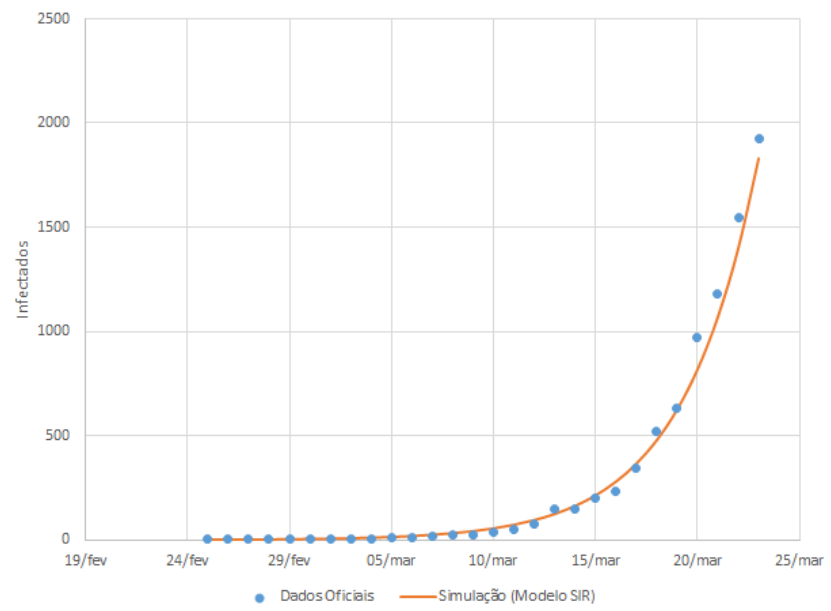

Figura 4: Comparação entre o número de infectados fornecido pelo modelo SIR com os dados oficiais no Brasil (25/02/2020 a 23/03/2020).

Fonte: Autores

A Figura 5 mostra a projeção da Covid-19 no Brasil, utilizando os parâmetros reportados na Tabela 1. Observa-se que o parâmetro $\alpha$ foi obtido no período em que medidas de isolamento social, fechamento de Universidades e Escolas, fechamento do comércio, entre outras, ainda não tinham sido impostas. Medidas de distanciamento social e de conscientização da população acerca dos sintomas da Covid-19 iniciaram-se por volta de 16 de março de 2020 (GONÇALVES, et al. 2020).

As curvas foram simuladas para um período de 180 dias a partir de 25 de fevereiro de 2020. O tempo $\mathrm{t}=0$, no gráfico apresentado na Figuras 5 , corresponde a data de 25/02/2020.

Com os dados iniciais de infectados no Brasil durante o período de 25/02/2020 a 23/03/2020 (BRASIL, 2020), o modelo projeta que o pico máximo da Covid-19 no Brasil ocorreria em 09 de maio de 2020, como pode ser observado na Figura 6. A curva foi simulada para um período de 180 dias a partir de 25 de fevereiro de 2020. $O$ tempo $t=0$, no gráfico apresentado na Figuras 6, corresponde a data de 25/02/2020. 


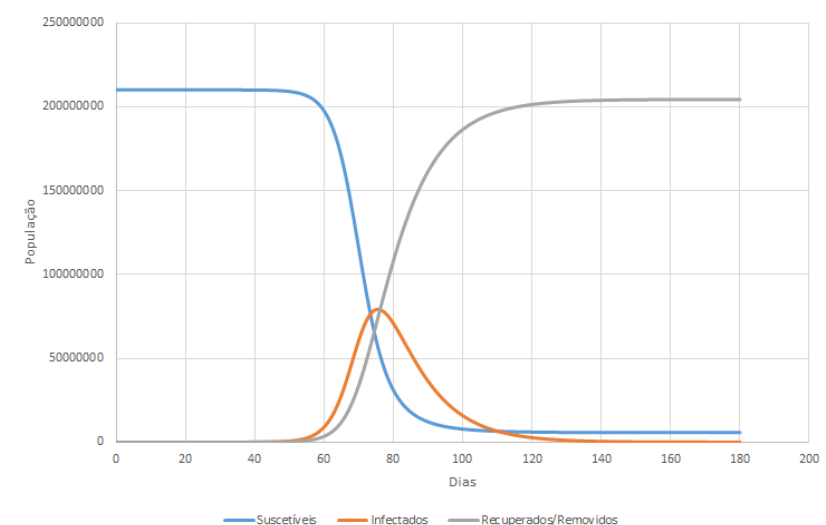

Figura 5: Simulação da evolução da Covid-19 no Brasil (projeção 1).

Fonte: Autores

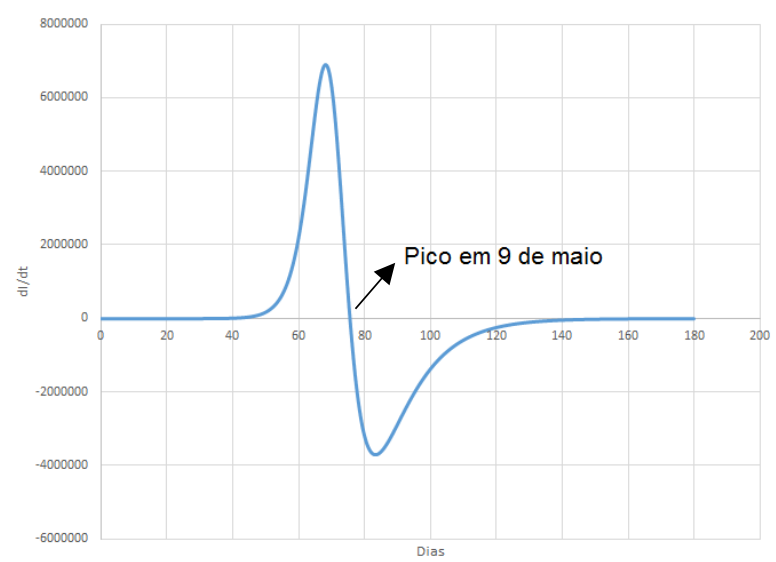

Figura 6: Taxa de variação da população infectada no Brasil (projeção 1).

Fonte: Autores

A Figura 7 mostra a comparação entre os dados simulados e os dados oficiais fornecidos pelo MS para os infectados no Brasil no período de 14/04/2020 a 03/05/2020, disponível em https://covid.saude.gov.br/ (acesso em 03/05/2020). Os resultados da simulação foram obtidos com os parâmetros dados na Tabela 2. O coeficiente de determinação obtido foi $\mathrm{R}_{\mathrm{d}}^{2}=$ 0.997826179 .

Neste período, a taxa de reprodução da doença é $\mathrm{R}_{0}=1.71891162$. Observase redução da taxa de reprodução quando comparado com a simulação anterior, o que mostra o efeito das restrições e medidas de distanciamento social. No entanto, o número de infectados continuou crescendo e a projeção do modelo foi de 151.578 casos em 08 de maio de 2020. Os dados oficiais 
nesta data foram de 145.328 casos no Brasil, o que corresponde a um erro relativo percentual de $4.3 \%$.

Tabela 2: Parâmetros usados no modelo SIR para Brasil

\begin{tabular}{|c|c|c|c|c|}
\hline \multicolumn{1}{|c|}{ Período } & População (N) & $\begin{array}{c}\mathrm{I}_{0}\left(\mathrm{n}^{\mathrm{o}} \text { de }\right. \\
\text { infectados } \\
\text { inicial }\end{array}$ & $\beta\left[\right.$ Dias $\left.^{-1}\right]$ & $\begin{array}{c}\alpha\left[\text { Dias }^{-1}\right] \\
\text { (Parâmetro } \\
\text { ajustado) }\end{array}$ \\
\hline $\begin{array}{c}14 / 04 / 2020 \mathrm{a} \\
03 / 05 / 2020\end{array}$ & 210.000 .000 & 25.262 & 0.1 & 0.171891162 \\
\hline
\end{tabular}

Fonte: Autores

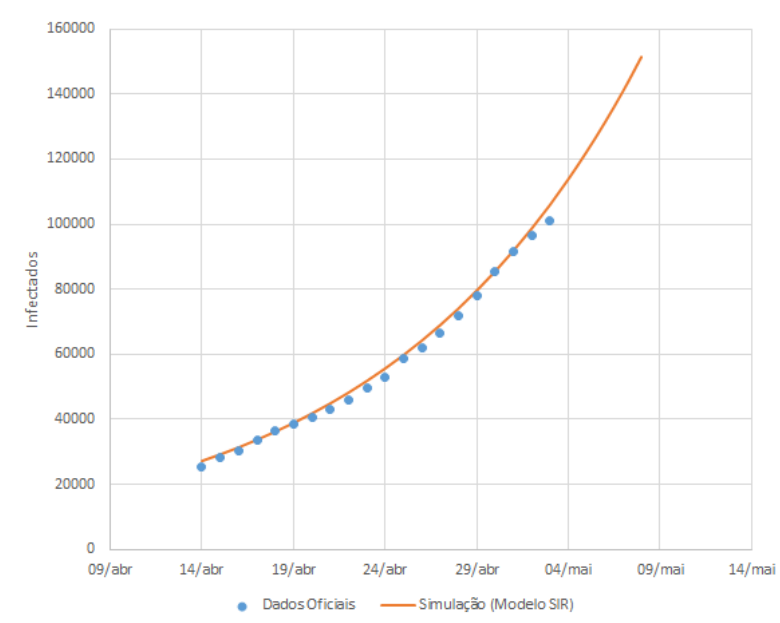

Figura 7: Comparação entre o número de infectados fornecido pelo modelo SIR com os dados oficiais no Brasil (14/04/2020 a 03/05/2020).

Fonte: Autores

A Figura 8 mostra a projeção da Covid-19 no Brasil, utilizando os parâmetros reportados na Tabela 2. As curvas foram simuladas para um período de 200 dias a partir de 14 de abril de 2020. $O$ tempo $t=0$ no gráfico corresponde a data de 14/04/2020. Quando comparada com a Figura 5, observa-se uma tendência de achatamento da curva de infectados e um deslocamento do pico máximo para agosto de 2020.

Considerando os dados de infectados no Brasil no período de 14/04/2020 a 03/05/2020, o modelo prevê uma estimativa para o pico máximo da Covid-19 no Brasil em 15 de agosto de 2020, como mostra a Figura 9. Isto é, no Brasil o número de casos começaria a decrescer (diminuir) em agosto. 


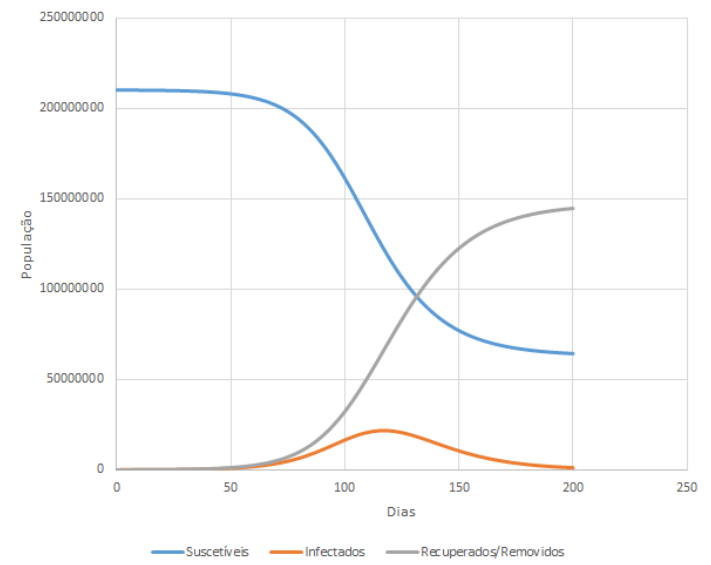

Figura 8: Simulação da evolução da Covid-19 no Brasil (projeção 2).

Fonte: Autores

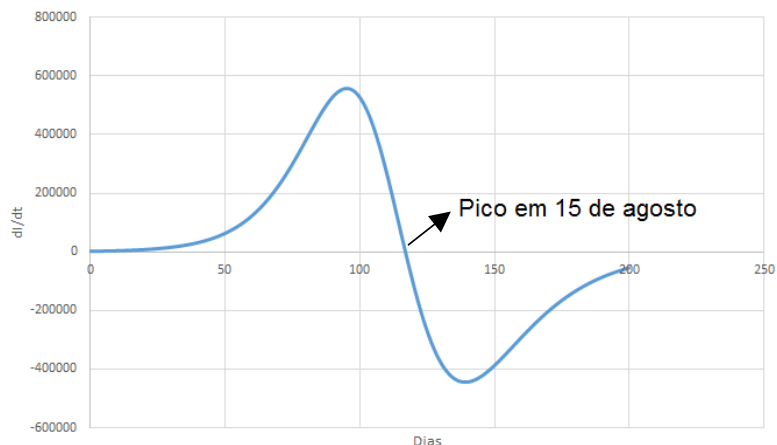

Figura 9: Taxa de variação da população infectada no Brasil (projeção 2).

Fonte: Autores

Destaca-se que este resultado é uma projeção do modelo SIR e, portanto, não é exato, principalmente para períodos mais distantes. Alerta-se também para os casos subnotificados. Em toda previsão matemática é importante ter dados reais e, na atual conjuntura, o número de infectados no Brasil pode ser muito maior do que o informado pelo MS. Por outro lado, a dinâmica da epidemia pode sofrer mudança causada pelo surgimento de uma vacina e/ou tratamento (medicamentos, etc) eficaz que reduza o seu avanço.

A Figura 10 mostra a comparação entre os dados simulados e os dados oficiais de infectados pela Covid-19 no Estado da Paraíba no período de 28/03/2020 a 03/05/2020 (BRASIL, 2020). Os parâmetros utilizados na simulação são apresentados na Tabela 3. Neste caso, o coeficiente de determinação obtido do ajuste do modelo foi $R_{d}^{2}=0.997$. 
Tabela 3: Parâmetros usados no modelo SIR para o Estado da Paraíba

\begin{tabular}{|c|c|c|c|c|}
\hline Período & População $(\mathrm{N})$ & $\begin{array}{c}\mathrm{I}_{0}\left(\mathrm{n}^{\mathrm{o}} \mathrm{de}\right. \\
\text { infectados } \\
\text { inicial }\end{array}$ & $\beta\left[\mathrm{Dias}^{-1}\right]$ & $\begin{array}{c}\alpha\left[\mathrm{Dias}^{-1}\right] \\
\text { (Parâmetro ajustado) }\end{array}$ \\
\hline $\begin{array}{c}28 / 03 / 2020 \mathrm{a} \\
03 / 05 / 2020\end{array}$ & 3.766 .528 & 14 & 0.1 & 0.223588607313835 \\
\hline
\end{tabular}

Fonte: Autores

Neste período analisado, a taxa de reprodução da doença é $R_{0}=2.236$. $A$ previsão projetada pelo modelo para o dia 08 de maio de 2020 foi de aproximadamente 2.170 casos de infectados na Paraíba. Os dados oficiais epidemiológicos no Estado da Paraíba, nesta data, foram divulgados com 2.030 casos de infectados, e o erro relativo percentual foi de $6.8 \%$.

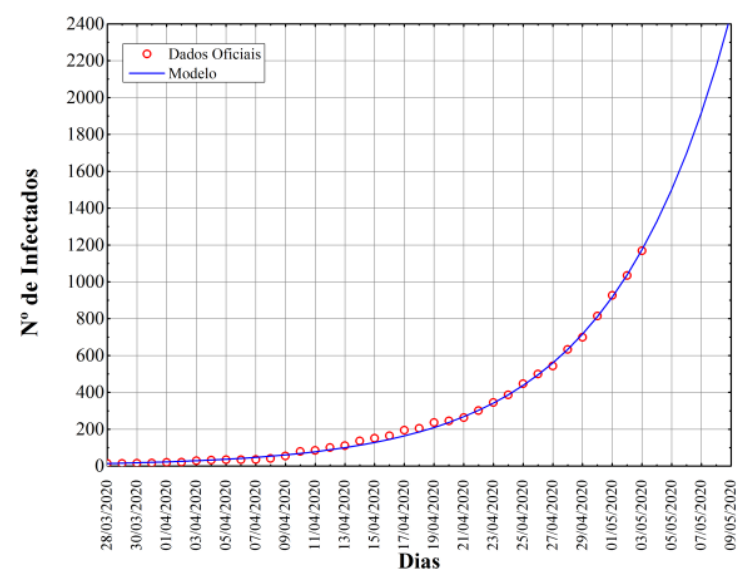

Figura 10: Comparação entre o número de infectados fornecido pelo modelo SIR com os dados oficiais no Estado da Paraíba (28/03/2020 a 03/05/2020).

Fonte: Autores

A Figura 11 mostra a projeção da Covid-19 no Estado da Paraíba, utilizando os parâmetros reportados na Tabela 3. Considerando os dados de infectados no Estado da Paraíba no período de 28/03/2020 a 03/05/2020, o modelo prevê uma estimativa para o pico máximo da Covid-19 em 8 de julho de 2020, como mostra a Figura 12. 


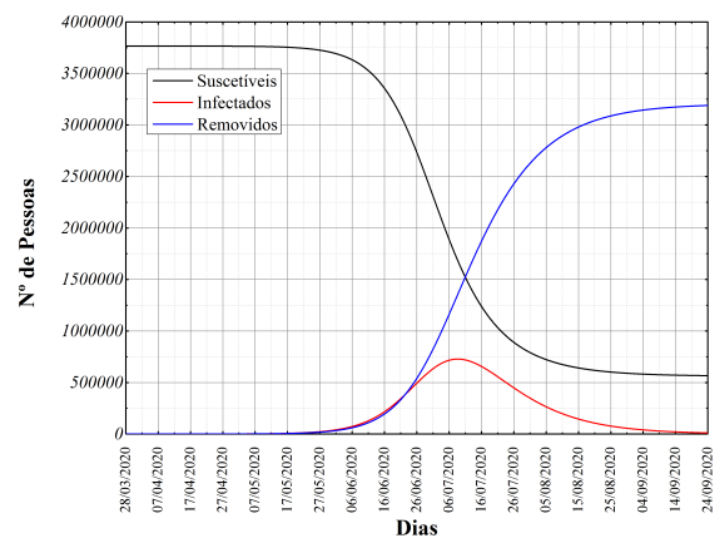

Figura 11: Simulação da evolução da Covid-19 no Estado da Paraíba.

Fonte: Autores

Destaca-se que este resultado é uma projeção do modelo e, portanto, não é exata, principalmente para períodos mais distantes. Além disso, acredita-se que a Paraíba é um dos Estados que menos testa e, consequentemente, apresenta a maior taxa de letalidade do país. Quando os dados não são próximos da realidade, os erros nas projeções e estimativas são aumentados.

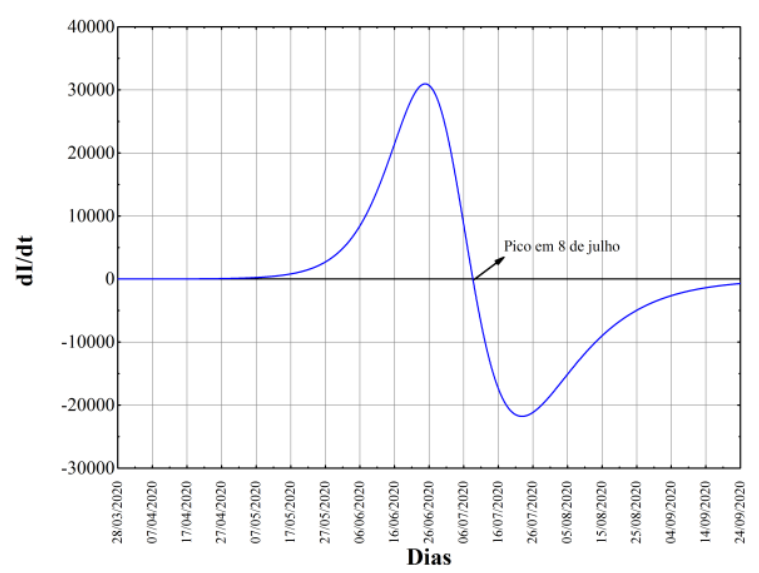

Figura 12: Taxa de variação da população infectada no Estado da Paraíba Fonte: Autores 


\section{Conclusão}

O modelo SIR utilizado nesse estudo é a sua versão mais simples. De acordo com os parâmetros estatísticos foi possível concluir que o Modelo SIR descreve bem os dados de infectados no Brasil e no estado da Paraíba para os períodos analisados. As projeções para períodos mais distantes podem não ser tão exatas (segundo a literatura o erro pode chegar a $50 \%$ ou mais) e o número de pessoas infectadas com a Covid-19 pode ser muito maior do que os casos notificados. Neste caso, para estabelecer estratégias ótimas para combater uma epidemia são necessários dados reais.

Neste trabalho, foi possível verificar o efeito das medidas de distanciamento social na taxa de reprodução $\left(\mathrm{R}_{0}\right)$ da doença. É possível inferir que a diminuição de $\mathrm{R}_{0}$ é devido as medidas de distanciamento social, já que não existe atualmente nenhuma vacina (diminuindo o número de pessoas suscetíveis) e/ou tratamentos eficazes que diminuam o tempo de recuperação, ou seja, essas medidas preventivas são atualmente as melhores formas para conter a epidemia, evitando um colapso no sistema de saúde do Brasil. Assim, recomendações da $\mathrm{OMS}^{1}$ (Organização Mundial da Saúde) são importantes para reduzir a taxa $\mathrm{R}_{0}$ para um valor menor que 1 , o qual caracteriza a erradicação da epidemia.

Por fim, no decorrer da epidemia, os parâmetros vão sendo melhor definidos uma vez que a característica da epidemia vai sendo identificada e variações dos modelos podem ser desenvolvidas. Por exemplo, seria possível usar modelagem matemática para fazer um controle ótimo da evolução da epidemia com o mínimo de isolamento social e protegendo o sistema de saúde. Neste caso, diferentes estratégias de isolamentos social seriam implementadas no decorrer do tempo, levando-se em conta a interação entre as cidades e sempre fixando a capacidade do sistema de saúde e maximizando a taxa de novos infectados.

\footnotetext{
${ }^{1}$ Lave as mãos com água e sabão ou higienizador à base de álcool. Mantenha pelo menos 1 metro de distância entre você e qualquer pessoa que esteja tossindo ou espirrando. Evite tocar nos olhos, nariz e boca. Cobrir a boca e o nariz com a parte interna do cotovelo ou lenço quando tossir ou espirrar (em seguida, descarte o lenço usado imediatamente). Fique em casa se não se sentir bem. Se você tiver febre, tosse e dificuldade em respirar, procure atendimento médico. Pessoas doentes devem adiar ou evitar viajar para as áreas afetadas por coronavírus. Os viajantes que retornam das áreas afetadas devem monitorar seus sintomas por 14 dias e seguir os protocolos nacionais dos países receptores; e se ocorrerem sintomas, devem entrar em contato com um médico e informar sobre o histórico de viagem e os sintomas (Organização Mundial da Saúde, 2020).
} 


\section{Referências}

BARROS, Aline Mide Romano de. Modelos matemáticos de equações diferenciais ordinárias aplicados à epidemiologia. Revista de Ciências Exatas e Tecnologia, Londrina, v. 2, n. 2, p. 62-67, ago. 2007.

BASSANEZI, Rodney Carlos; FERREIRA JÚNIOR, Wilson Castro. Equações diferenciais com aplicações. São Paulo: HARBRA, 1988.

BRASIL, MINISTÉRIO DA SAÚDE. Coronavírus (COVID-19). Brasília, 2020. Disponível em: <<http://covid.saude.gov.br/>>. Acesso em: 03 de mar. de 2020.

GONÇALVES, Glênio Aguiar et al. A evolução epidêmica do COVID-19 Modelo SIR. Pelotas, 9 abr. 2020. Disponível em: $<$ https://wp.ufpel.edu.br/fentransporte/2020/04/09/a-evolucao-epidemica-docovid-19-modelo-sir>>. Acesso em: 2 maio 2020.

GRUBER, A. Covid-19: o que se sabe sobre a origem da doença? Disponível em: $\quad<<h t t p s: / / j o r n a l . u s p . b r / a r t i g o s / c o v i d 2-o-q u e-s e-s a b e-s o b r e-a-o r i g e m-d a-$ doenca/ >>. Acesso em 08 de junho de 2020.

LUIZ, Mônica Helena Ribeiro. Modelos Matemáticos em Epidemiologia. 2012. Dissertação (Mestrado Profissional em Matemática) - Universidade Estadual Paulista, Rio Claro, 2012.

LYRA, Wladimir et al. COVID-19 pandemics modeling with SEIR $(+\mathrm{CAQH})$, socialdistancing, and age stratification. The effect of vertical confinement and release in Brazil, 2020. Doi: https://doi.org/10.1101/2020.04.09.20060053.

MA, Zhien; ZHOU, Yicang; WU, Jianhong. Some Recent Results on Epidemic Dynamics Obtained by Our Group. [S. I.]: World Scientific Publishing Company, 2009.

MANRIQUE-ABRIL, Fred G. et al. Modelo SIR de la pandemia de COVID-19 en Colombia. Revista de Salud Pública, Bogotá, v. 22, n. 01, p. 1-9, mar. 2020. 
MORRISON, Rebecca E.; CUNHA, Americo. Embedded model discrepancy: a case study of zika modeling.: A case study of Zika modeling. Chaos: An Interdisciplinary Journal of Nonlinear Science, [s.I.], v. 30, n. 5, p. 051103, maio 2020.

OLIVEIRA, Elida; ORTIZ, Brenda. Ministério da Saúde confirma primeiro caso de coronavírus no Brasil. Brasília: G1, 26 fev. 2020. Disponível em: $<$ https://g1.globo.com/ciencia-e-saude/noticia/2020/02/26/ministerio-da-saudefala-sobre-caso-possivel-paciente-com-coronavirus.ghtml >. Acesso em: 1 maio 2020.

ORGANIZAÇÃO MUNDIAL DA SAÚDE. Surto de Doença de Coronavírus (COVID-19): Diretrizes para o Público. Suiça, 2020. Disponível em: <<https://www.who.int/es/emergencies/diseases/novel-coronavirus-2019/advice - for-public >>. Acesso em: 3 maio 2020.

ROCHA, Diana Isabel Cardoso. Modelos matemáticos aplicados à epidemiologia. 2012. Dissertação (Mestrado em Métodos Quantitativos em Economia e Gestão) - Universidade do Porto, Porto, 2012.

TAVARES, João Nuno. Modelo SIR em epidemiologia. Revista de Ciência Elementar, Porto, v. 5, n. 2, p. 1-12, 30 jun. 2017.

Wu, F., Zhao, S., Yu, B. et.al. Um novo coronavírus associado a doenças respiratórias humanas na China. Nature 579, 265-269 (2020). https://doi.org/10.1038/s41586-020-2008-3. 